\title{
The Revolution of Entrepreneurship Through E- Commerce Model: Questionnaire-based Research in China
}

\author{
Juntao Li* \\ Bellevue High School, 1201 96th Ave SE, Bellevue, WA, USA \\ *Corresponding author: Juntao Li, plibhs2022@gmail.com
}

\begin{abstract}
E-commerce is evolving as an innovative tool in a wide range of areas, and has changed the face of entrepreneurship by making them more efficient and dynamic. In this paper, the author firstly reviewed the current development of e-commerce and entrepreneurship, as well as existing opportunities and challenges in this field. This paper also combines both qualitative method (literature review) and quantitative method (questionnaire-based research) to explore the impact of e-commerce on the landscape of entrepreneurship. The findings of this study suggest that the development of e-commerce has triggered fundamental changes in entrepreneurial activities. It significantly promotes the entry of new start-ups and facilitates the growth of their businesses. Meanwhile, small- and medium-sized firms also encounter greater challenges when they try to adopt and implement e-commerce.
\end{abstract}

Keywords: E-commerce; Entrepreneurship; Business strategies; Literature review; Questionnaire

Publication date: September 2021; Online publication: September 30, 2021

\section{Introduction}

Nowadays, job markets are already close to the saturation point with a reduced number of job opportunities and a fairly good stock of graduates ${ }^{[1]}$. Coupled with the boom in e-commerce and the prevailing use of the Internet, people are more inspired to jump into the world of entrepreneurship globally. With everything going online, e-commerce is a great platform for entrepreneurs to start their own business or to make their business expand globally. This paper sets out to investigate the effects of e-commerce on entrepreneurship in China, which has provided a unique and particularly suitable context for academic discussions. On the one hand, China is unarguably the world's largest e-commerce market, which grows at an annual rate of $120 \%$ since 2003 and shows no sign of stopping ${ }^{[2]}$. On the other hand, entrepreneurial activities are thriving in recent years in China. According to Global Entrepreneurship Monitor, China's total early-stage entrepreneurial activity index in 2019 is 8.70, a relatively higher figure compared with other developing countries ${ }^{[3]}$. This indicates a need to understand the various perceptions of how e-commerce impacts entrepreneurship in China, and shed light on future research directions.

This paper is organized as follows: Section 2 elaborates the framework for literature review and introduces the academic point of view of the impact of e-commerce on entrepreneurial business models. Section 3 includes research methodology and questionnaire responses used for empirical analysis. Section 4 summarizes the whole paper with key findings. 


\section{Literature Review}

\subsection{Captions or numbering}

In this increasingly globalized and intensively competitive world, it has become an irreversible trend for ecommerce and entrepreneurship to play central roles in the economy of any size. As e-commerce has acquired enormous development in past years, it also brings in various opportunities for entrepreneurs to set up their own business or to open up new revenue with an enhanced competitive edge. This mix of ecommerce and entrepreneurship also attracts the academic community to reflect further on their contributions. Therefore, this section conducts a systematic literature review of existing academic studies, and provides a better understanding of current academic progress.

Figure 1. represents the process of extracting the full set of academic articles, papers, and journals. To begin with, this paper retrieved all the relevant literature by searching keywords "e-commerce" and "entrepreneur" in Scopus and Web of Science database, which are considered the major academic databases to obtain documentation resources. Our database research has returned 1157 publications in total. Going forward, we excluded those articles that we are unable to get full access to and identified repetitive articles by checking titles, abstracts, and authors. Next, we further filtered these publications based on full-text reading, content review, and theme analysis using Microsoft excel to identify those stick closely to our research topic. At last, we defined the scope of literature analysis with a total of 1161 relevant studies.

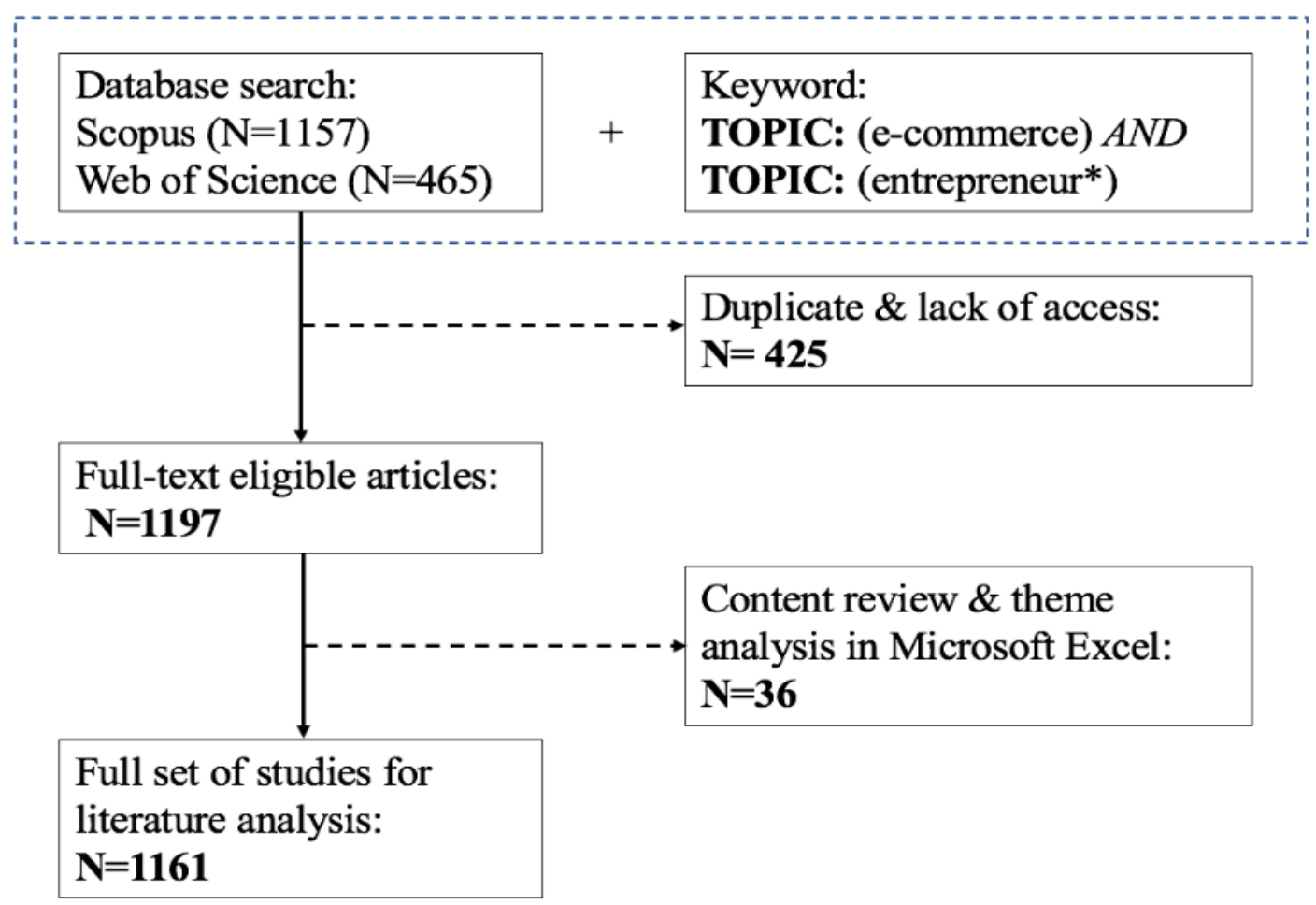

Figure 1. Selection Process for Literation Analysis

\subsection{Literature Analysis}

In the final examination, this paper took a deep look at the selected 1161 studies to grasp their central notions and academic value. As can be seen from Figure 2., the number of publications focused on ecommerce and entrepreneurship goes up year by year, and peaked in 2019 with 162 published results. This fact indicates that the mix of these two areas has generated extensive interest in the academic community, and this trend is very likely to grow with the rapid development of technological innovations and the widespread use of e-commerce applications. 


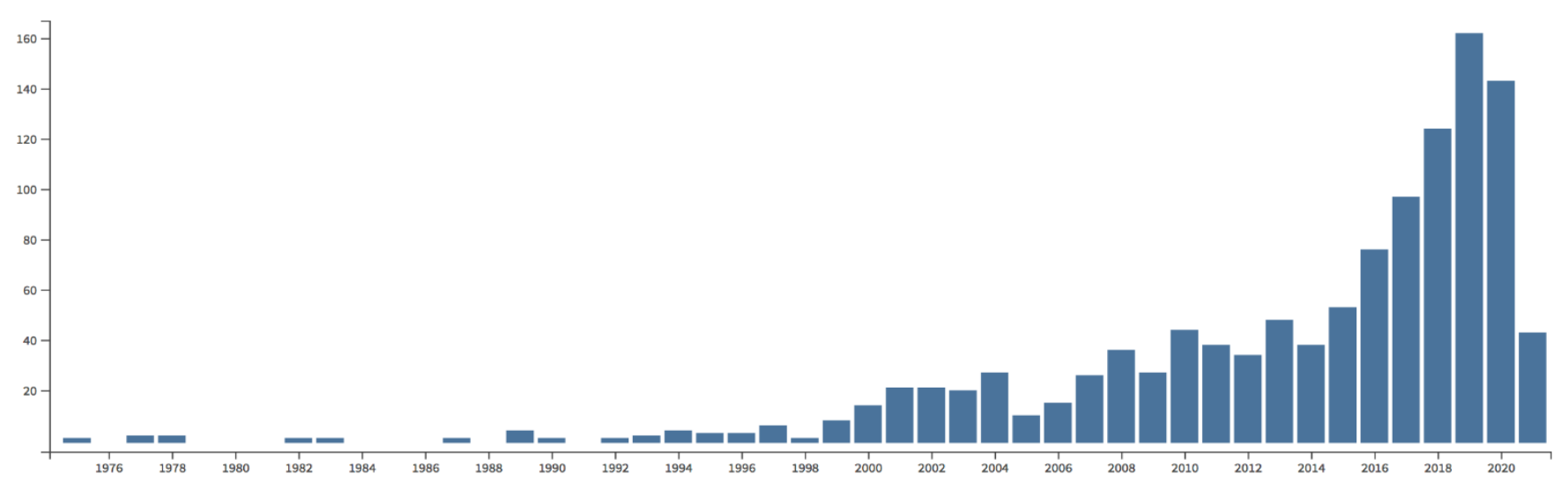

Figure 2. Documents by Year

Meanwhile, this paper also used a treemap to depict the distribution of the subject area as shown in Figure 3. A majority of literature pertains to the impact of e-commerce and entrepreneurship on business and economic development. Notably, 126 studies are related with education or educational research, especially the successful implementation of entrepreneurship education in universities. When looking at documents by country or region, as shown in Figure 4., we can notice that the USA, China, and the UK are the top three countries with the most publications, taking up nearly $58 \%$ of the total.

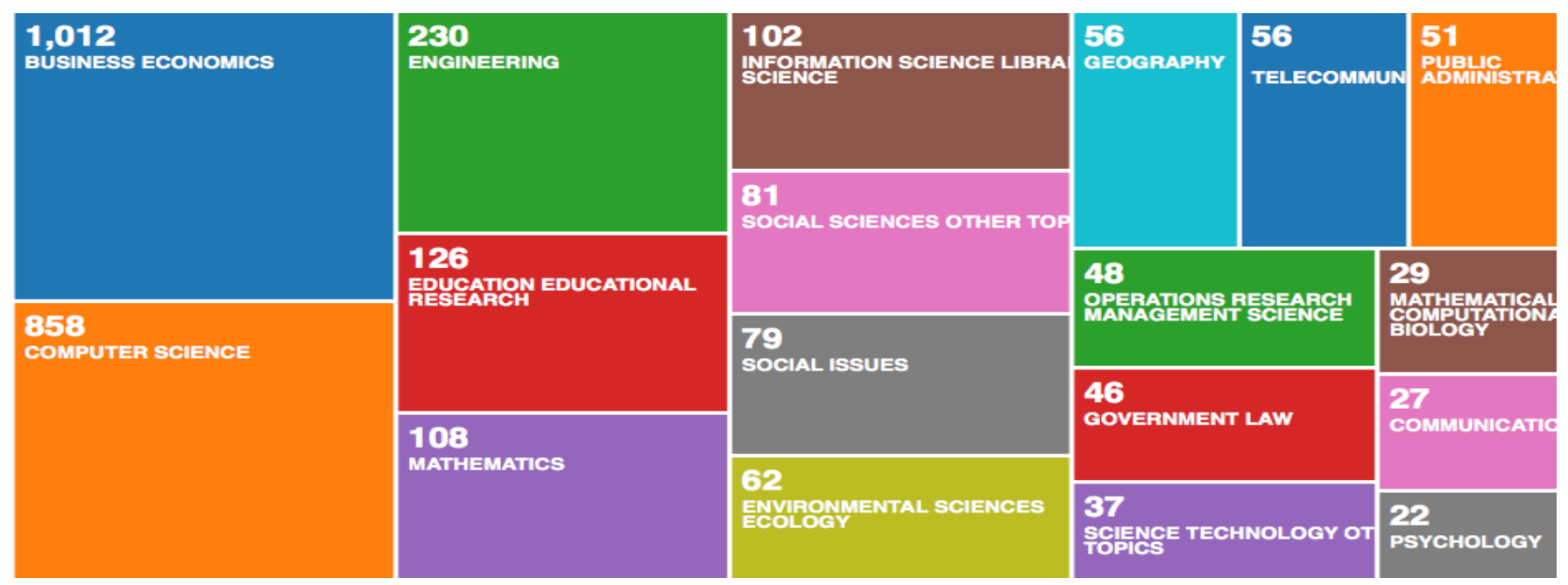

Figure 3. Documents by Research Area

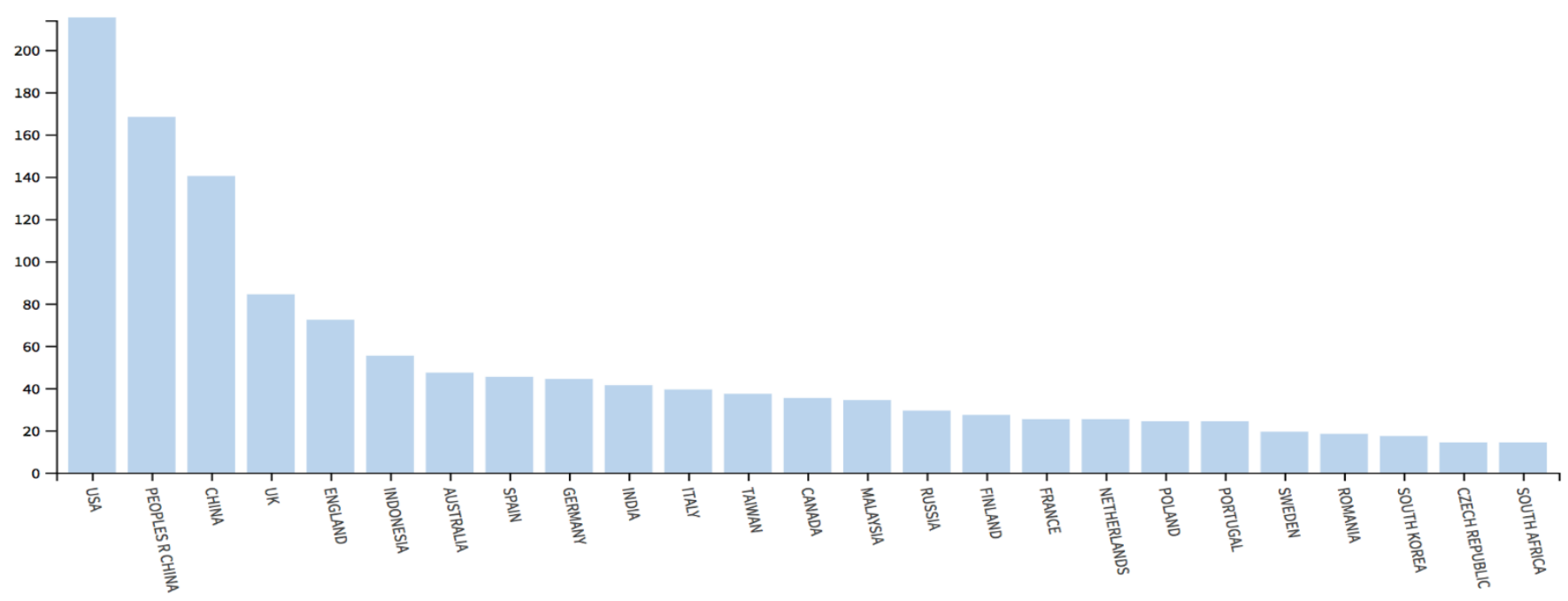

Figure 4. Documents by Country or Territory 
This paper also reviewed the full set of literature by creating their citation report, which reflects citations sourced from all databases. The summary statistics are presented in Table 1. The h-index measures the comprehensive impact of an author's research output or academic performance by counting the number of his or her published, highly impactful papers. The h-index here is 42 , which means that there are 42 publications that have been cited 42 times or more. The average citation per item is 6.43 , a relatively higher value when compared to other studies over the same period. The citation report provides further proof of the far-reaching impact in the field of e-commerce and entrepreneurship, as well as their advanced progress in the academic circle.

Table 1. Citation Report

\begin{tabular}{cc}
\hline Title & Value \\
\hline h-index & 42 \\
Average citation per item & 6.43 \\
Sum of times cited & 7,465 \\
Citing articles & 6,541 \\
\hline
\end{tabular}

After a careful review of their content, this paper also found out that the role of e-commerce in transforming entrepreneurship displayed distinct patterns in developed and developing countries. For developed countries with the more mature capital market and advanced technology applications, ecommerce has become a principal instrument for small- and middle-sized entrepreneurial (SME) companies, entailing them a multitude of competitive advantages to rival big corporations, such as to attract new clients in global market, promote their products and services, and smarter ways to finalize payment and distributions. For example, Lefebvre et al. piloted a study in Canadian SME firms in the manufacturing sector, and identified their evolutionary trajectories to adopt e-commerce ${ }^{[4]}$. Their findings validated the business benefits derived from the accumulative and self-enhancing nature of e-commerce, and also pointed out how these SMEs utilized the power of the Internet and websites to operate and expand the business. Similarly, after scrutinizing relevant studies in United States, Australia, Denmark, and Sweden, there is an obvious trend that with an excellent basis of entrepreneurship activities, the adoption of e-commerce could empower the existing business by creating their technological edges.

In most developing countries, the transformation of entrepreneurship using e-commerce tends to develop slowly, especially when compared to those in more developed regions. However, previous studies have found out that the power of e-commerce can open doors of opportunity for individual businesses and private enterprises, who once had no choice but stuck to traditional commercial mode, and this effect is particularly noticeable in rural areas. For instance, Kabongo et al. conducted their study in Congo to find out the correlation between e-commerce adoption and entrepreneurship. Their analysis further showed that SMEs in the telecommunications sector have made the most use of e-commerce, and this newly added entrepreneurial feature was conducive for small business growth ${ }^{[5]}$. Another paper published by Asian Development Bank Institute (ADBI) evaluated the regional development of e-commerce across rural and urban areas in China and quantitatively measured its impact on entrepreneurship. Their findings revealed that as e-commerce continues to progress, there is a remarkable increase in the entry of local start-ups, especially for household entrepreneurship in the sector of manufacturing and wholesale ${ }^{[6]}$.

However, although the academic community acknowledges numerous benefits that come along with the boom of e-commerce, it also inevitably presents the landscape of entrepreneurship with several challenges. One notable phenomenon is that in new waves of e-commerce and technical innovations, the exit of incumbent businesses has also increased due to a generally low level of organizational capacity and 
a lack of technological readiness among small- and middle-sized entrepreneurial enterprises ${ }^{[7]}$. A study in Nigeria indicated that local start-ups are still struggling to fully grasp the potential of e-commerce solutions to expand their business, and the progress was far behind when compared with other Internet-intensive countries ${ }^{[8]}$. On the other hand, since the adoption of e-commerce is mostly dominated by big companies, the growing competition between small businesses and larger firms has speeded the obsolescence of those who cannot catch up with the latest advancements in technology, including but not limited to online advertising, mobile applications, and omnichannel sales model.

\section{Methodology}

From our literature analysis, we came to know that e-commerce is evolving as one of the most disruptive forces in driving the reinvention of entrepreneurship, and bringing the world both opportunities and challenges to its sustained growth. Hereby, this paper seeks to investigate the specific role of e-commerce in the field of entrepreneurship, assess both the benefits and risks when combining e-commerce and entrepreneurship, and offer some critical insights into creating successful start-ups or achieving corporate transformations using e-commerce.

\subsection{Research Methods}

Many researchers have adopted the method of the questionnaire and analyzed the collected results to examine the specific effects of e-commerce on entrepreneurship. Following this most common procedure used in previous literature, this research was exploratory and interpretative in nature, taking the form of the questionnaire and qualitative assessment among all the surveyed start-up companies. This hybrid approach has various attractive features. Firstly, it captures the complexities of each entrepreneurial firm in the context of e-commerce, and allows us to delve into the micro-enterprise environment and firm-level perspectives on the market, industry, customers, and technology. Secondly, since the landscape of entrepreneurship still lacks a complete set of quantitative metrics, qualitative methods can be more useful for identifying e-commercial factors that influence entrepreneurship and characterizing their distinctive features.

\subsection{Data Collection}

\subsubsection{Study Subjects}

Since this paper aims to distribute our questionnaire among new start-ups and entrepreneurial companies in China, the study subjects are directly retrieved from the Enterprise Innovation and Entrepreneurship Database, which is mainly sponsored by the Institute of Social Science Survey, Peking University ${ }^{[9]}$. This project is widely known for its annual Enterprise Survey for Innovation and Entrepreneurship (ESIEC) in China, and combines the collected results into an integrated and searchable national database, covering 31 provincial regions and nearly all industry categories.

To ensure the timeliness and accuracy of data, this paper selects start-ups that were registered during the period from 2012 to 2017 (the most recent survey data in ESIEC), and there is a total of 6.1 million enterprises nationwide. This paper narrowed down the scope by excluding those companies that have not or have not yet started e-commerce strategies, those with inactive business activities in the past five years, and those with incomplete contact information. Considering the large volume of data and heavy workload to research all the potential subjects, this paper also used the method of stratified sampling based on region density. Thus, we obtained a list of 2042 entrepreneurial firms as final study subjects for our large-scale survey.

\subsubsection{Questionnaire Design}

The questionnaire was designed to investigate the impact of e-commerce on the landscape of 
entrepreneurship. It took the form of both ten-point Likert scale questions and open-ended questions that allows participants to evaluate e-commercial effects from multiple aspects. The first type of questions set out to gather background information about the enterprise, including the size of the company, number of staff, the type of business, the company's development over these years, whether it has a website, and whether it is supported by venture capital. This section mainly focused on the mission and vision of each entrepreneurial company, inquiring into its history, culture, and structure, as well as its current market performance, business objectives, and future growth strategies.

The second type of question is more relevant to the utilization of e-commerce, e-commercial technologies, e-commerce platforms, or any extent of their use. This section can help understand the type of e-commerce being used and on what kind of activities, as well as the rationale behind their business objectives and e-commercial applications. And the third type of question is centred around the opportunities and challenges that these enterprises are facing in the process of using e-commerce. This paper has listed a series of opportunities and challenges refined and sorted out from our preliminary research. For each question participants were asked to respond to each question using a 10-point Likert scale, ranging from " 0 " denoting "don't agree" to " 1 " representing "totally agree". There are also open-end questions for respondents to fill out what efforts are underway to overcome the said challenges.

The questionnaires were distributed directly through the contact information of 2042 selected entrepreneurial companies in ESIEC, we withdrew a total of 1619 copies of effective questionnaire for further analysis.

\subsection{Result Analysis}

\subsubsection{Background of Using E-commerce Model}

As shown in Table 2., most of our research subjects are small-sized and middle-sized companies. Judging from their market value, nearly all these companies are in the seed stage or growing stage, which exhibits high growth of potentiality.

Table 2. Company Size

\begin{tabular}{cc}
\hline Company size (Number of employees) & Number of companies \\
\hline $10-49$ & 271 \\
$50-299$ & 643 \\
$300-499$ & 465 \\
$500+$ & 240 \\
\hline
\end{tabular}

When looking at the geographical distribution of these 1619 valid questionnaires, this paper found out that most entrepreneurial companies are located on the southeast coast of China. Guangdong province owns the highest number of start-ups, and $14.86 \%$ of enterprises were registered there. Jiangsu and Zhejiang provinces follow not far behind, accounting for $8.83 \%$ and $7.97 \%$, respectively. This distribution is noted to be in accord with the prosperity of e-commerce in China. According to the e-commerce development index in China by province, e-commerce sellers are centralized in the Yangtze River Delta and Pearl River delta for their highly-developed commercial basis, especially in Guangdong, Zhejiang, and Jiangsu provinces ${ }^{[10]}$. This phenomenon indicates that entrepreneurial firms located in provinces or cities where businesses flourish are more likely to take advantage of the e-commercial benefits. Meanwhile, the pie chart below shows the breakdown of industry distribution based on responses to questionnaires. $49 \%$ of companies are in the retail industry and occupy the largest share of the e-commerce market, suggesting a wide use of e-commerce technologies in retail. E-commerce applications also extend to a broad range of 
different industries, including personal care and services, finance, logistics, and cross-border trade.

\section{Industry distribution}

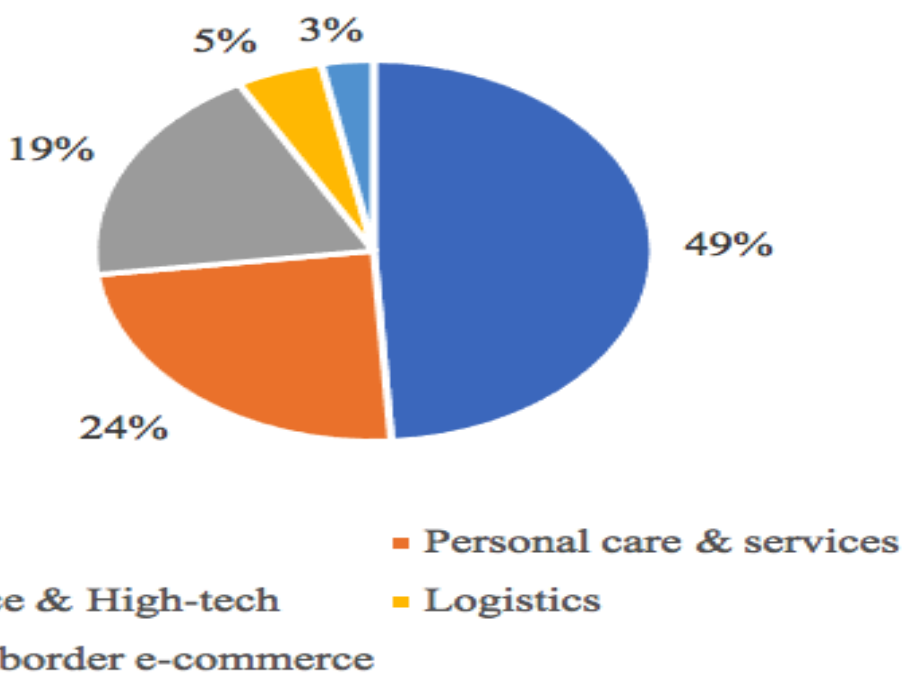

- Retail

- Finance \& High-tech

- Cross-border e-commerce
- Personal care \& services

Logistics

Figure 5. Industry Distribution of Surveyed Companies

\subsubsection{Opportunities and Challenges When Using E-commerce Model}

The second and third section of questions provide us with a clearer and more intuitive picture of how ecommerce revolutionize the landscape of entrepreneurship. For opportunities, e-commerce serves as an efficient alternative for brick-and-mortar stores. More than $80 \%$ of start-up companies replied that it waives a majority of the complicated documentation process and eases out huge investments in the initial stage. As a result, there are $89.01 \%$ of entrepreneurs or business owners who would take this chance to shift from employment to entrepreneurship. Therefore, it can be concluded that e-commerce has significantly reformed entrepreneurial minds and made the business model more dynamic. Furthermore, $74.10 \%$ of companies graded 8 and above on the operational cost efficiency of e-commerce. Paperless office and workfrom-home mode allow them to lower administrative expenses. $44.03 \%$ of responses, mainly from relatively large-sized companies, felt that using e-commerce could free them from geographical limits and expand their businesses to the global market, especially for those in the retail, logistics, and cross-border trade industry. Benefits associated with market competitiveness are not apparent as we expected, with only $8.46 \%$ believed that e-commerce would help them become more competitive. Responses also indicate that e-commerce giants, such as Amazon, Walmart, and Alibaba, can drive newcomers out of the online retailer market.

Meanwhile, e-commerce also introduced multiple risks as it lowers the market barriers to entry and thus intensifies the competition. More than half of respondents considered that web development, technology procurement, and digital marketing can be expensive, and keeping up with the latest technical trends is mostly capital-intensive and out of the reach of these entrepreneurial companies. Another critical challenge is that despite their strongest desire to move to e-commerce practices, almost $58 \%$ of companies said that they were in shortage of qualified technical people to oversee the e-commerce operations and create the customization that they needed. Lastly, nearly $11 \%$ of companies were highly concerned with cybersecurity, which has scared off several start-ups from testing that water. These responses reflect that there are still large gaps in their business goals and current e-commercial capacities. 


\title{
OPPORTUNITIES
}

\author{
$120.00 \%$ \\ $100.00 \%$ \\ $80.00 \%$ \\ $60.00 \%$ \\ $40.00 \%$ \\ $20.00 \%$ \\ $0.00 \%$
}

Chart Area

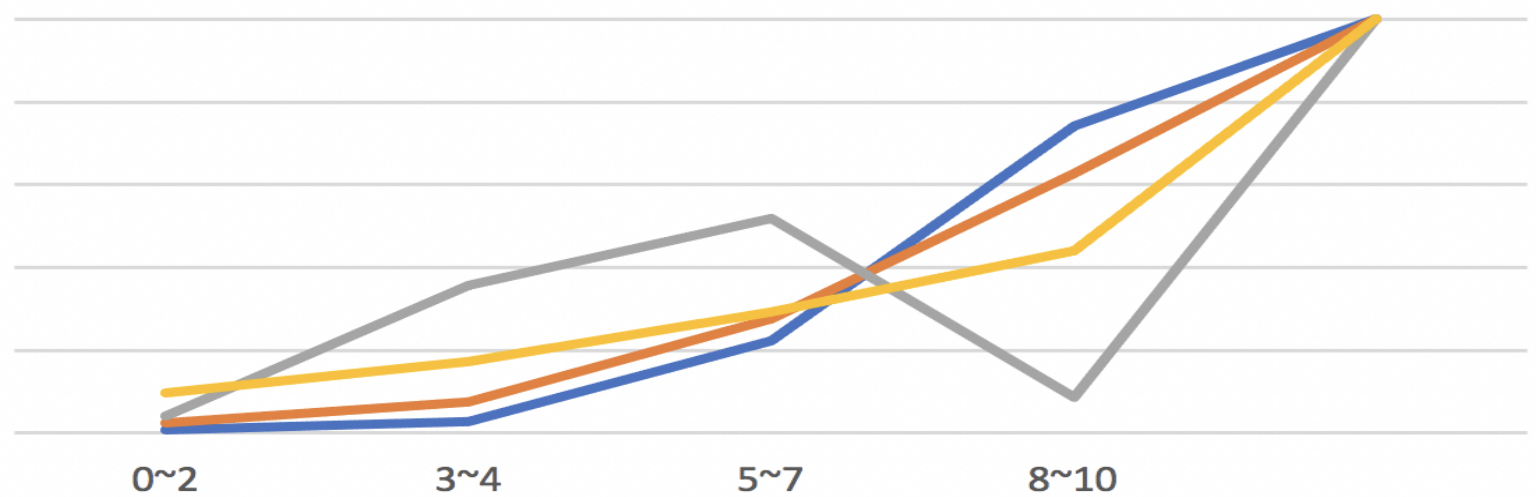

Investment cost savings

Operational cost efficiency

Market competitiveness

Global outreach \& participation

Figure 6. Responses on Opportunities

\section{CHALLENGES}

\begin{abstract}
$120.00 \%$
$100.00 \%$

$80.00 \%$

$60.00 \%$

$40.00 \%$

$20.00 \%$

$0.00 \%$
\end{abstract}

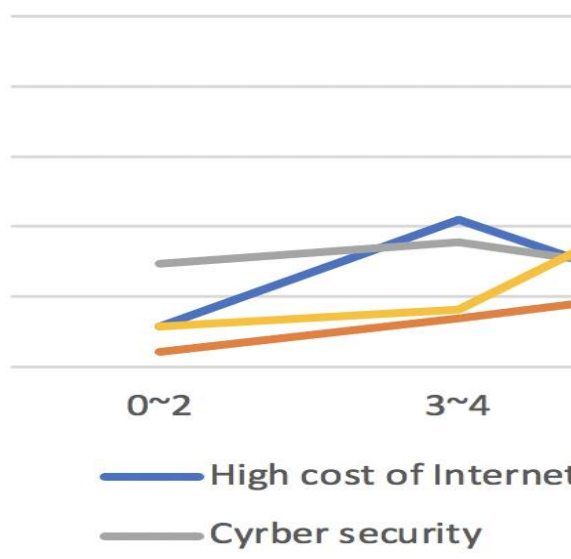

Figure 7. Responses on Challenges

\section{Conclusions}

The main goal of this paper is to explore the impact of e-commerce on entrepreneurial activities through a combination of both qualitative and quantitative analyses. In the questionnaire-based research, this study has identified that e-commerce has brought about dramatic changes to the landscape of entrepreneurship. It stimulates the start-up of new companies and businesses, and opens up fresh opportunities, including investment cost savings, operational cost reduction, and global outreach. However, our findings also show that challenges, such as the high cost of the Internet, lack of technical expertise, and competitive marketing strategies, still remain, and there is still a long way for entrepreneurial firms to fully grasp the advantages of e-commerce.

\section{Disclosure statement}

The author declares no conflict of interest. 


\section{References}

[1] "Significance of E-Commerce for Entrepreneurs Today - Loyal Determined," Loyaldetermined.com, 2020. Available: https:/loyaldetermined.com/significanceof-e-commerce-for-entrepreneurs-today/. M. Ben Rabha, M.F. Boujmil, M. Saadoun, B. Bessaïs, Eur. Phys. J. Appl. Phys.

[2] J. Ho, F. Poh, J. Zhou and D. Zipser, China consumer report 2020 - McKinsey, 2020. [Online]. Available: https://www.mckinsey.com/featured-insights/china/china-consumer-report-2020-themany-faces-of-the-chinese-consumer. [Accessed: 15- Jun- 2021].

[3] GEM Global Entrepreneurship Monitor, 2021. [Online]. Available: https://www.gemconsortium.org/data. [Accessed: 15- Jun- 2021].

[4] L. Lefebvre, É. Lefebvre, E. Elia and H. Boeck, "Exploring B-to-B e-commerce adoption trajectories in manufacturing SMEs", Technovation, vol. 25, no. 12, pp. 1443-1456, 2005. Available: 10.1016/j.technovation.2005.06.011.

[5] J. D. Kabongo and J. O. Okpara, "ICT possession among Congolese SMEs: an exploratory study", Journal of Small Business and Enterprise Development, vol. 21, no. 2, pp. 313-326, 2014. Available: 10.1108/jsbed-10-2013-0143.

[6] X. He, 2009, “The Development of Entrepreneurship and Private Enterprise in The People's Republic of China And its Relevance to Transitional Economies," Journal of Developmental Entrepreneurship, 14(01): 39-58. Available: 10.1142/s1084946709001132.

[7] A. Tang, 2019, “A Systematic Literature Review and Analysis on Mobile Apps in M-Commerce: Implications for Future Research," Electronic Commerce Research and Applications, 37: 100885. Available: 10.1016/j.elerap.2019.100885.

[8] H. Awa, O. Ojiabo and B. Emecheta, 2015, "Integrating TAM, TPB and TOE Frameworks and Expanding Their Characteristic Constructs for E-Commerce Adoption by SMEs," Journal of Science \& Technology Policy Management, 6(1): 76-94. Available: 10.1108/jstpm-04-2014-0012.

[9] Institute of Social Science Survey, Peking University, Isss.pku.edu.cn, 2021. Available: http://www.isss.pku.edu.cn/xwzx/1320594.htm.

[10] C. Briefing, 2021, "China's 46 New Cross-Border E-Commerce Zones: A Brief Primer," China Briefing News, 2021. Available: https://www.china-briefing.com/news/china-unveils-46-newcross-border-e-commerce-zones-incentives-foreign-investors-faqs/. 